\title{
Yard Crane Scheduling in the Presence of Uncertain Handling Times
}

\author{
Hamid Ghaani Farashahi ${ }^{1}$, Yong $\mathrm{Wu}^{2}$ and Peter Tatham ${ }^{3}$
}

\begin{abstract}
This paper researches the problem of the scheduling of yard cranes within a yard block inside a container port terminal. Existing models found within the literature generally consider improvements to the scheduling of yard crane operations using a static and deterministic approach where all the problem parameters are fixed or certain. In reality, however, due to the interaction between the yard cranes working in a given yard block, the vast number of decisions required to be made in a short timeframe, as well as the unexpected events and failures that occur in real-world scheduling environment, there is a degree of uncertainty in the operation of such yard cranes. Hence, in this paper, the handling time of yard cranes is assumed to be uncertain and that it follows a triangular distribution. The resulting challenge of scheduling under uncertainty is NP-hard and presents a complex optimization problem. This research develops a heuristic solution by considering the real yard crane operational constraints like fixed safety distance, inter-crane interference, and simultaneous storage and retrieval operations. The proposed heuristic approach is able to find near optimal solutions in reasonable time. The performance of the proposed approach is demonstrated through multiple experiments which are carried out on a large set of instances which reflect different operational scenarios.
\end{abstract}

Keywords: container terminal, yard crane scheduling, uncertain handling time

\section{Introduction}

Container terminals are an essential component of today's global trade as evidenced by data from the United Nations Conference on Trade and Development (UNCTAD, 2014) which indicates that global containerized trade has doubled from 80 million 20 -foot equivalent units (TEUs) shipped in 2002 to some 160 million TEUs a decade later. In parallel, world container port throughput has increased over the same period and reached over 651 million TEUs movements in 2013 (UNCTAD, 2014).

Unsurprisingly, this growth in containerization leads to increasing management challenges including the interactions between different terminal operations and the associated equipment. In order to stay competitive, terminal operators must attempt to optimize the allocation of resource and scheduling of equipment, with the clear benefits of improving ship turnaround time, handling a greater number of containers, enhancing service levels, reducing the service cost, reducing handling operations dwell-times, and reducing energy consumption.

$1 \quad$ PhD Candidate, Griffith Business School, Griffith University, Gold Coast campus, QLD 4222, Australia, E-mail: hamid.ghaanifarashahi@griffithuni.edu.au

2 Senior Lecturer, Griffith Business School, Griffith University, Gold Coast campus, QLD 4222, Australia, E-mail: yong.wu@griffith.edu.au

3 Professor, Griffith Business School, Griffith University, Gold Coast campus, QLD 4222, Australia, E-mail: p.tatham@griffith.edu.au 
The key resources in a typical container terminal are divided into three different categories: Quay Cranes (QCs), Yard Cranes (YCs), and Yard Trucks (YTs). Once a vessel arrives at the nominated berth, containers are usually unloaded from a vessel using one or more QCs which move containers from the vessel to YTs. The YTs then move the containers to their next location where YCs will offload the containers into an area of the terminal known as a yard block. This process is reversed for containers that are to be loaded onto the vessel. The overall process is summarized in Figure 1.

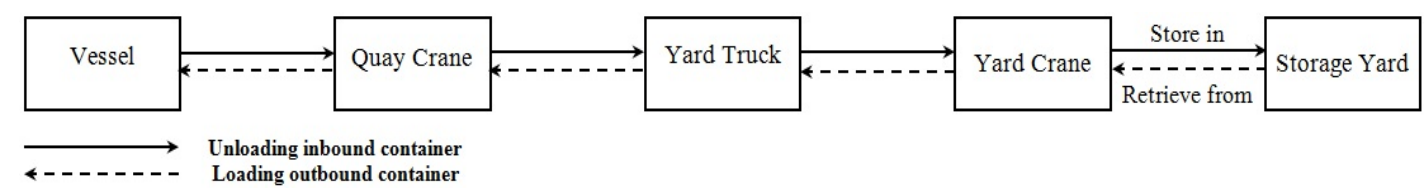

Figure 1. Typical Container Flows in a Container Terminal

Steenken et al. (2004) noted that minimizing the turnaround time of vessels is the main objective of container terminal operations. In order to achieve this, the allocation and scheduling of berths, QCs, YCs, and YTs need to be the focus of research aimed at developing improved models and, where possible, reducing the equipment idle time. Over recent years, the speed of QCs and YCs has improved considerably, although, the performance of both QCs and YCs are mutually dependent. QCs are mounted on a railway and just move along the wharf, but YCs have to cover a large container yard to store and retrieve containers. The maximum speed of typical YC is between approximately a half and one-third of a typical QC (Ng and Mak, 2005b; Murty, 2007). Thus, in order to keep a QC working at or near its full capacity, some 2-3 YCs are required to support each QC (Petering et al., 2009). Hence, YC operations play a key role in improving the overall container terminal performance and failure to optimize the sequence of YC movements has the clear potential to cause a bottleneck within the terminal.

Due to the nature of the storage moves which begin when the QCs start discharging the incoming vessel, they are not allowed to take place earlier than their target time which is usually derived from QC working schedules. However, minimizing lateness for storage jobs only leads to an improved utilization of YTs. On the other hand, minimizing lateness for retrieval jobs improves the QCs and, hence, berth performance. As a result, retrieval jobs have a higher priority in comparison to storage jobs. In reality, container terminals do not operate like clockwork, rather they involve the inter-dependent operation of multiple processes and this causes a wide range of uncertainty in the resultant container handling/travelling times and lowering/hoisting times. Figure 2 shows a typical yard crane operation where a container is unloaded off a yard truck. 


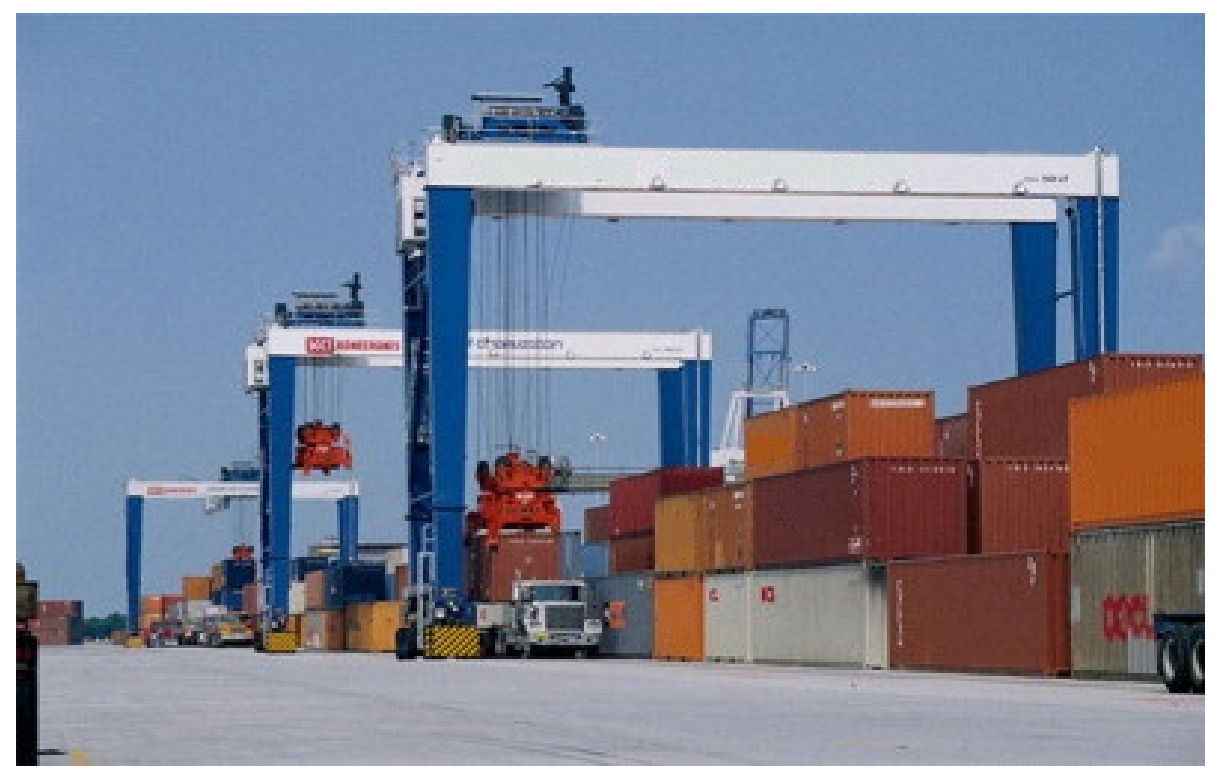

Figure 2 . A Yard Crane Interacting with a Yard Truck

In this paper, the problem of YC scheduling to handle both storage and retrieval moves with different target times simultaneously is presented. In doing so, a number of realistic operational constraints that are imposed by multiple yard cranes are considered including inter-crane interference among YCs, the safety distance between adjacent YCs, and uncertainty in $\mathrm{YC}$ handling times.

\section{Literature Review}

As outlined above, obtaining a smooth and continuous flow of containers needs significant coordination between the different types of equipment operating in the terminal. Unsurprisingly, therefore, the issue of container terminal improvement has attracted a significant volume of research that is focused on the associated decision processes, resource allocation and scheduling. Specifically, Narasimhan and Palekar (2002) note that finding an optimal solution for single yard crane dispatching problem is NP-hard, which means it is unlikely to be possible to find such an optimal solution in polynomial time. To cope with this challenge, a number of mathematical models, heuristic approaches and simulations have been proposed under different settings. For example, Murty et al. (2005) studied the different operations at a container terminal and presented a decision support system designed to achieve high efficiency and, hence, a competitive global terminal. Subsequently, Chen et al. (2007) studied the coordination among different types of container handling equipment and demonstrated their impact on the overall terminal efficiency.

Several studies have focused on the deployment and scheduling of one or more YCs in container yard. Linn et al. (2003) introduced a formal procedure for deploying YCs within a storage yard including cross gantry moves. Ng and Mak (2005a) studied the problem of scheduling a single YC carrying out a given set of jobs with different ready times in order to optimize the total job waiting time. Jung and Kim (2006) introduced the pickup scheduling problem of outbound containers taking simultaneous multiple YCs and multiple QCs into consideration. These authors assumed that interference would occur among YCs and therefore introduced a safety distance between adjacent YCs, but these authors also assumed that the work schedules for QCs are given. In comparison, the real time multiple YC scheduling problem has been studied by Chen and Langevin (2011) through considering loading operations and linear gantry movements in order to minimize the completion time 
of the handling of all the outbound containers. Sharif and Huynh (2012) argue that maximizing YC utilization and minimizing YTs waiting time are the main objectives of YC scheduling.

Scheduling YCs in a single block with reference to inter-crane interference, the safety distance between two YCs, and using both storage and retrieval moves has been researched by Li et al. (2009) who presented a discrete time MILP model. They developed heuristics and a rolling-horizon algorithm, and the resultant data suggested that their proposed methods are more efficient than previous models. In the subsequent research, Li et al. (2012) extended their earlier study through consideration of continuous time MILP model to reach better computational times and quality solutions.

However, despite the fact that there are many uncertain factors in real time container terminal operations, only a few scholars have taken uncertainty into consideration in container terminal environment. That said, the berth and QC allocation problem with uncertain arrival time and loading/unloading of ships was addressed by Zhou and Kang (2008) and a genetic algorithm (GA) was proposed to overcome the problem. In a similar study, Han et al. (2010) incorporated the discrete berth allocation and QC assignment problem simultaneously through consideration of stochastic vessel arrival and container handling time parameters. Their work was able to generate robust solutions by the use of a simulation based genetic algorithm search. The job assignment problem for Automated Guided Vehicle (AGV) in an automated container terminal was consider by Angeloudis and Bell (2010) who took uncertainty in AGV service time into account. Escudero et al. (2013) studied the YT movement between the pick-up at container terminal and delivery at a consignment point under uncertain transit times due to congestion. Lu and Le (2014) addressed the integrated scheduling of loading and unloading of QCs, YTs, and YCs with a certain driving distance for every YT but uncertainty in YT travel rate, YC speed and hoisting/lowering operation time, although these authors did not consider safety distances and inter-crane interference.

Thus, to our best knowledge, the problem of YC scheduling with uncertainty and incorporating safety distances to avoid inter-crane interference has not been addressed by scholars. With this in mind, this paper focuses on YCs scheduling among a single yard block, with uncertain handling time and safety distances and offers solutions that are believed to be close to the optimum.

\section{Proposed Heuristic Method}

As indicated above, the problem of YC scheduling is NP-hard; hence, to reach near optimal solutions, a heuristic method has been developed. In this paper such heuristic is presented in order to carry out the collection of unscheduled storage and retrieval containers by YCs within a given block with respect to safety distance, inter-crane interference, and uncertain handling time.

To achieve this, the first stage is to determine which YC will perform the movement of a given container. This is achieved by the use of the $K$-means clustering algorithm (Hartigan, $1975)$, where the number of clusters $(K)$ is assumed to be equal to the number of YCs in a block.

The main idea adopted in the proposed heuristic method is to obtain an initial schedule solution using a fixed handling time. As discussed earlier, the retrieval jobs have more priority, so they are scheduled first. After scheduling all retrieval containers, storage jobs are inserted into the resultant schedule one by one. When all containers are scheduled and in order to obtain an initial feasible schedule which remains robust with respect to any changes in the YC handling times, the generated solution is checked for containers that may cause 
overlapping by introducing a buffer time. The detailed flow of the proposed method is presented as follows to generate an initial schedule:

- Step 1: Finding Sequence of Containers for each Yard Crane

In this step, a job sequence for each YC is generated. In this study, YC handling time is assumed to be the mean of a triangular distribution with parameters (1.2, 2.0, and 3.4) minutes. As both types of containers (storage/retrieval) may exist in the YC job list, the first task is to separate all retrieval jobs for each YC and insert them into a job list, then sort them based on proposed target time in ascending order. For each job in the list, the finish time is calculated with respect to start time is assumed to be equal to the target time. Once the calculation of finish time for each job is complete, it is possible to identify the feasible and unfeasible jobs from the beginning to the end of the list. A job is feasible when its finish time is equal to or less than the start time of next job. If there are any unfeasible jobs in the list these jobs are re-scheduled. To achieve this, all jobs are checked one by one from beginning to the end of the list until the first unfeasible job is found, then the search continues until the first feasible job is detected, and then all jobs between these two points are marked as a group of unfeasible jobs. Without changing the sequence of jobs, unfeasible jobs are then postponed or advanced as necessary so that they can be achieved in a feasible time, with the selection being based on the creation of better performance. This procedure continues until all retrieval jobs become feasible.

- Step 2: Insert Storage Jobs

After all retrieval jobs have been scheduled, the storage jobs are considered. All remaining storage jobs are inserted into a temporary list and are sorted based on their target time. The storage jobs are inserted into the schedule generated by step 1 one at a time. The earliest storage job is taken out from the temporary list, and we find the first available time in the YC job list after its target time which has enough room to perform this job. If the proposed start time does not overlap with the adjacent crane job list, the start time is fixed for this storage job. If this condition cannot be met, the search is continued until this storage job can be inserted into the YC job list without having an overlap.

- Step 3: Predictive Overlapping Jobs

To deal with uncertain YC handling time, Step 3 predicts which containers may cause overlapping in the face of uncertainty and resolves this problem. To achieve this, a buffer time is inserted before and after jobs which are located in the overlapping area in order to absorb the uncertainty. The buffer time is equal to the difference between maximum YC handling time (3.4 minutes) and the amount of handling time which is used to generate the initial schedule. This buffer time recognizes both the uncertainty in the earliness of start time as well as delays in the handling time of containers in the overlapping area that may lead to overlapping in the presence of uncertainty.

This step is applied to two adjacent YCs simultaneously. It starts from the beginning of the YC job list to find any job in the overlapping area of the crane and inserts a buffer time between them. If the addition of this buffer time leads to an overlap, the corresponding job in the neighbouring crane will be found. This results in two jobs in two adjacent YCs that may cause an overlap in the face of uncertainty. To resolve the problem, overlapping jobs are moved to one of these two YCs and a new schedule is generated from first step. The new schedules are rejected if any of these two jobs still causes overlapping with the inserted buffer time. If both new schedules are accepted, the one with smaller objective function will be retained. In the case that both schedules are rejected, we attempt to find a new position for the overlapping jobs using their original YCs and choose a new schedule with a smaller objective function. This step continues until all jobs are checked. 


\section{Numerical Experiments}

The research problem described in this paper is similar to Li et al. (2009) and Li et al. (2012), and therefore the objective function which was proposed by them is used to validate the proposed method. The main aim of this paper is to optimize YC operations in the face of uncertainty in a way that will minimize the linear combination of Storage lateness $(S l)$, Retrieval earliness $(R e)$, and Retrieval lateness $(R l)$. As discussed earlier, handling retrieval jobs has more priority than storage jobs, so in the objective function the retrieval lateness weight $\left(w_{r l}\right)$ is set to ' 2 ' and the weights for storage lateness and retrieval earliness are set to ' 1 '.

$$
\text { Minimize : } \quad w_{r l} \sum_{j=1}^{n} R l_{j}+w_{s l} \sum_{j=1}^{n} S l_{j}+w_{r e} \sum_{j=1}^{n} R e_{j}
$$

To validate the effectiveness of the proposed method with uncertain YC handling times, a triangular distribution with parameters 1.2, 2.0, and 3.4 minutes is used to generate random handling times for each job. To check the robustness of the proposed method, we conduct simulation runs using the schedules generated. We strictly follow the job sequence in the schedules but use random numbers generated from the triangular distribution to represent the handling times. Due to the introduction of these random numbers, the start and finish times of jobs might need to be adjusted accordingly. First, the consecutive jobs on the initial schedule are grouped together. Jobs belong to the same group when the finish time of the current job is equal to the start time of next job. Then the start time and finish time for all jobs are re-calculated using generated random handling times. In each group the start time of first job does not change if it is more than finish time of previous job, but the start and finish time of other jobs in the group are modified based on start time of previous job, job type, and uncertain handling time. In this stage, the containers sequence obtained by proposed method is not changed.

The proposed method is implemented using MATLAB R2014a (Version 8.3) on a PC Intel Core i5, 3.2 GHz with 8GB RAM to examine the numerical instances. Ten test scenarios were conducted to validate the performance of the proposed method. These test scenarios consist of different container operations: (1) only container storage, (2) only container retrieval, and (3) a mix of storage and retrieval.

Each test scenario is iterated 20 times to ensure that we can observe how the generated schedules perform under different set of random numbers (i.e., handling times). Table 1 shows the specification of the test scenarios and summarizes the computational results. The average of the objective function, storage lateness, retrieval earliness, and retrieval lateness were calculated by taking the mean over 20 replications for each test scenario. In all of the 200 simulation runs, only 2 instances (Scenario 10) were not possible to stick to the originally schedule generated, which means that the schedule would need to be adjusted on the fly in real world operations. The remaining instances handled the uncertain handling time satisfactorily which, in turn, indicates the proposed method is robust and reliable in the presence of uncertainty. 
Table 1. Test Scenarios Specification and Computational Results

\begin{tabular}{|l|l|l|l|l|l|l|l|l|}
\hline Scenario & Jobs & Slots & $\begin{array}{l}\text { Job } \\
\text { Type }\end{array}$ & $\begin{array}{l}\text { Objective } \\
\text { (minutes) }\end{array}$ & $\begin{array}{l}\text { Storage } \\
\text { Lateness }\end{array}$ & $\begin{array}{l}\text { Retrieval } \\
\text { Earliness }\end{array}$ & $\begin{array}{l}\text { Retrieval } \\
\text { Lateness }\end{array}$ & Unfeasibility \\
\hline 1 & 47 & 40 & R & 137.92 & 0 & 77.18 & 30.37 & $0 / 20$ \\
\hline 2 & 31 & 60 & R & 42.27 & 0 & 19.61 & 11.33 & $0 / 20$ \\
\hline 3 & 56 & 60 & R & 149.37 & 0 & 105.43 & 21.97 & $0 / 20$ \\
\hline 4 & 25 & 40 & S & 11.69 & 11.69 & 0 & 0 & $0 / 20$ \\
\hline 5 & 28 & 60 & S & 29.99 & 29.99 & 0 & 0 & $0 / 20$ \\
\hline 6 & 57 & 60 & S & 90.32 & 90.32 & 0 & 0 & $0 / 20$ \\
\hline 7 & 27 & 40 & R\&S & 31.90 & 6.85 & 11.59 & 6.73 & $0 / 20$ \\
\hline 8 & 55 & 40 & R\&S & 257.36 & 190.59 & 0.73 & 33.02 & $0 / 20$ \\
\hline 9 & 32 & 60 & R\&S & 25.32 & 17.35 & 6.19 & 0.89 & $0 / 20$ \\
\hline 10 & 53 & 60 & R\&S & 278.74 & 163.04 & 23.78 & 45.96 & $2 / 20$ \\
\hline
\end{tabular}

Figure 3 illustrates three job diagrams for 55 jobs in a 40-slot block with two YCs. Each rectangle is a symbol of one container move. A coloured rectangle with a solid line represents a scheduled container, and a shaded rectangle with dotted line represents a container move at its target time. Each coloured rectangle has three labels which indicate the assigned YC number, the job type ( $\mathrm{S}$ for storage and $\mathrm{R}$ for retrieval), and the job number respectively. All shaded and coloured rectangles have the same width which is equal to safety distance between YCs which, in common with the work of previous authors, is set at 8 slots, but the rectangle heights are different and this represents the container's variable handling time. Figure 3a illustrates the initial schedule generated by the proposed method with fixed handling time; from this it is easy to see that all jobs are feasible and that there is no overlapping job. Figures $3 b$ and Figure $3 c$ present two different sets (i.e., two simulation runs) of uncertain handling time on the same schedule. We can observe that, as we introduce uncertainty into container handling time, the schedules look different and the impact of uncertainty on the final schedule is evident. However, they still follow the same sequence generated in Figure 3a. The buffer time allocated for conflict-prone jobs handles the potential conflict quite well in these two cases.

\section{Conclusion}

The problem of YC scheduling under uncertainty has been presented in this paper and, in doing so, it closes a gap in the relevant literature. YC scheduling is one of the most important problems in a container terminal and one which directly impacts the terminal's performance. In this paper, the handling time of YCs is considered to have a stochastic value that follows a triangular distribution. The proposed method generates a smooth and fast solution as demonstrated in the above results. It is anticipated that further studies will consider the integration of scheduling of container terminal handling equipment such as QCs, YTs, and YCs with uncertain variables and realistic operational constraints to generate more reliable and high quality solutions. 


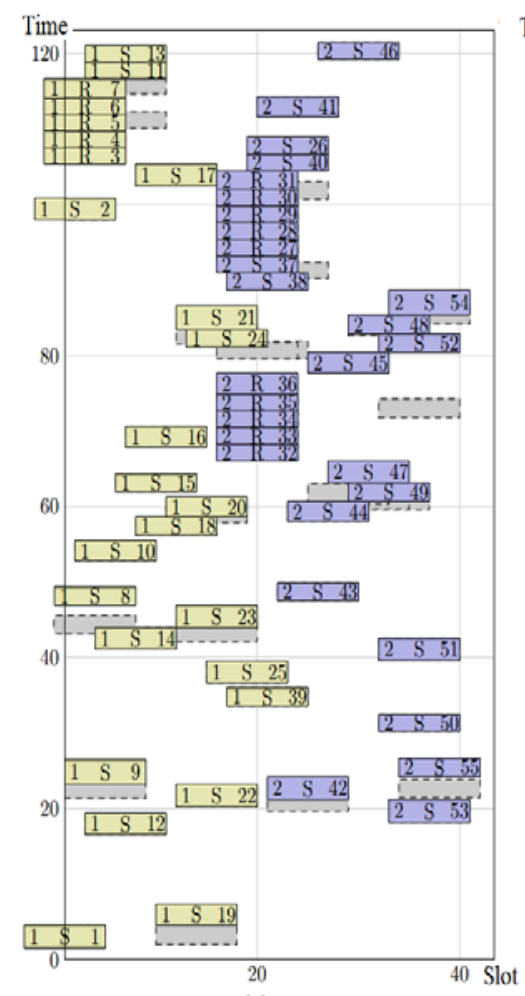

(a)

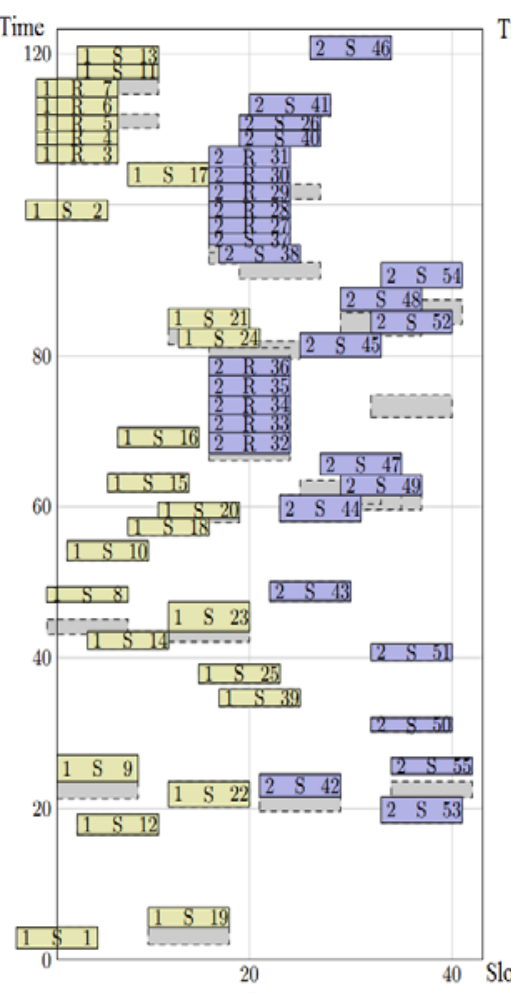

(b)

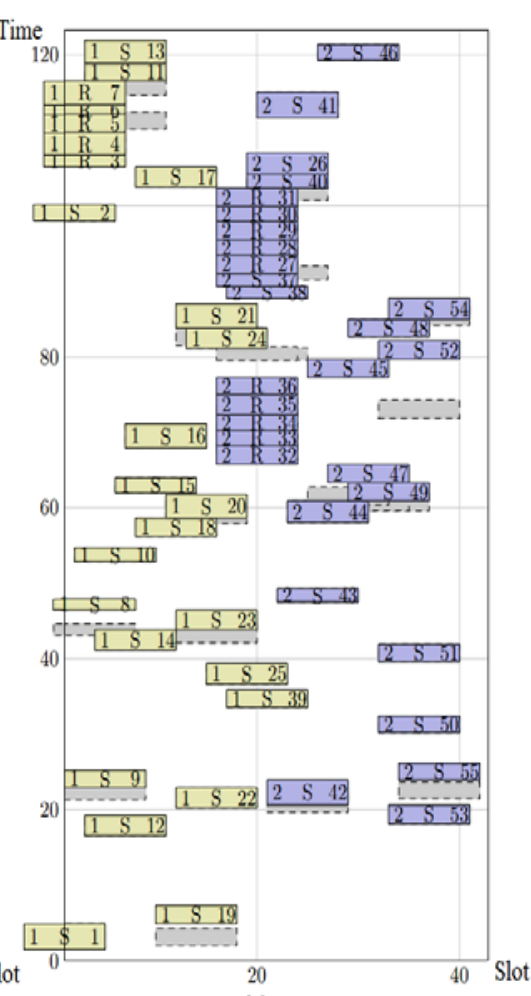

(c)

Figure 3. Initial Schedules Generated by Proposed Method with Fixed Handling Time (a), and Final Schedule with Variable Handling Time (b and c)

\section{References}

Angeloudis, P., and Bell, M. G. H. 2010. An uncertainty-aware AGV assignment algorithm for automated container terminals. Transportation Research Part E: Logistics and Transportation Review, 46(3), 354-366.

Chen, L., Bostel, N., Dejax, P., Cai, J., and Xi, L. 2007. A tabu search algorithm for the integrated scheduling problem of container handling systems in a maritime terminal. European Journal of Operational Research, 181(1), 40-58.

Chen, L., and Langevin, A. 2011. Multiple yard cranes scheduling for loading operations in a container terminal. Engineering Optimization, 43(11), 1205-1221.

Escudero, A., Muñuzuri, J., Guadix, J., and Arango, C. 2013. Dynamic approach to solve the daily drayage problem with transit time uncertainty. Computers in Industry, 64(2), 165-175.

Han, X.-l., Lu, Z.-q., and Xi, L.-f. 2010. A proactive approach for simultaneous berth and quay crane scheduling problem with stochastic arrival and handling time. European Journal of Operational Research, 207(3), 1327-1340.

Hartigan, J. A. 1975. Clustering algorithms. New York: John Wiley \& Sons, Inc.

Jung, S., and Kim, K. 2006. Load scheduling for multiple quay cranes in port container terminals. Journal of Intelligent Manufacturing, 17(4), 479-492.

Li, W., Goh, M., Wu, Y., Petering, M. E. H., de Souza, R., and Wu, Y. C. 2012. A continuous time model for multiple yard crane scheduling with last minute job arrivals. International Journal of Production Economics, 136(2), 332-343. 
Li, W., Wu, Y., Petering, M. E. H., Goh, M., and Souza, R. d. 2009. Discrete time model and algorithms for container yard crane scheduling. European Journal of Operational Research, 198(1), 165-172.

Linn, R., Liu, J.-y., Wan, Y.-w., Zhang, C., and Murty, K. G. 2003. Rubber tired gantry crane deployment for container yard operation. Computers \& Industrial Engineering, 45(3), 429-442.

Lu, Y., and Le, M. 2014. The Integrated Optimization of Container Terminal Scheduling with Uncertain Factors. Computers \& Industrial Engineering, 75(0), 209-216.

Murty, K. G. 2007. Yard crane pools and optimum layouts for storage yards of container terminals. Journal of Industrial and Systems Engineering, 1(3), 190-199.

Murty, K. G., Liu, J., Wan, Y.-w., and Linn, R. 2005. A decision support system for operations in a container terminal. Decision Support Systems, 39(3), 309-332.

Narasimhan, A., and Palekar, U. S. 2002. Analysis and algorithms for the transtainer routing problem in container port operations. Transportation Science, 36(1), 63-78.

Ng, W. C., and Mak, K. L. 2005a. An effective heuristic for scheduling a yard crane to handle jobs with different ready times. Engineering Optimization, 37(8), 867-877.

Ng, W. C., and Mak, K. L. 2005b. Yard crane scheduling in port container terminals. Applied Mathematical Modelling, 29(3), 263-276.

Petering, M. E. H., Wu, Y., Li, W., Goh, M., and de Souza, R. 2009. Development and simulation analysis of real-time yard crane control systems for seaport container transshipment terminals. OR Spectrum, 31(4), 801-835.

Sharif, O., and Huynh, N. 2012. Yard crane scheduling at container terminals: A comparative study of centralized and decentralized approaches. Maritime Economics and Logistics, 14(2), 139-161.

Steenken, D., Voß, S., and Stahlbock, R. 2004. Container terminal operation and operations research - a classification and literature review. OR Spectrum, 26(1), 3-49.

UNCTAD. 2014. Review of Maritime Transport. New York and Geneva: United nations conference on trade and developmen.

Zhou, P.-f., and Kang, H.-g. 2008. Study on berth and quay-crane allocation under stochastic environments in container terminal. Systems Engineering - Theory \& Practice, 28(1), 161-169. 